\title{
Der isoelektrische Punkt des Menschen-, Kuh-, Ziegen-, Hunde- und Meersehweinchenmilcheaseins.
}

\author{
Von \\ Arvo Ylppö (Helsingfors). \\ (Aus dem Kaiserin Auguste Victoria-Haus zur Bekämpfung der säuglingssterblich- \\ keit im Deutschen Reiche [Direktor: Prof. Leo Langstein] und aus dem Biolo- \\ gischen Laboratorium des Städtischen Krankenhauses Am Urban [Berlin], unter \\ der Leitung von Prof. Leonor Michaclis.)
}

(Eingegangen am 24. Mai 1913.)

Es gibt eine große Reihe von Untersuchungen, die sich mit der Frage beschäftigt haben, ob verschiedene Tierarten verschiedene Caseine haben. Fin Teil der Autoren verneint die Frage, ein anderer bejaht sie. Wir wollen von den ersteren nur z. B. Burow ${ }^{\mathbf{1}}$ ) erwähnen. Er hat durch Elementaranalysen keine sicheren Unterschiede zwischen den Caseinen der Kuh-, Kaninchen- und Frauenmilch finden können.

Langstein und Edelstein ${ }^{2}$, die später mehrere auf verschiedene Weise dargestellte reine Frauenmilcheaseinpräparate analysiert haben, haben dagegen immer einen bedeutend geringeren Phosphorgehalt als im Kuhmilcheasein gefunden. Durch diesen Befund haben sie einen Beweis erbracht, ,daß das Frauenmilchcasein und Kuhmilchcasein verschiedene Körper sind, wenn auch die Verschiedenheit vielleicht nur quantitativer Natur ist (geringere Menge der phosphorhaltigen Gruppe)".

Wir verzichten hier auf die nähere Besprechung der diesbezüglichen Literatur und weisen auf den Artikel: Caseine von R. W. Raudnitz in Sommerfelds Milchkunde 1909, S. $163 \mathrm{ff}$., auf die Zusammenstellung von Samuely im Biochem. Handlexikon von Abderhalden, Bd. IV, 1. Hälfte, S. 103, 1910, und auf die oben zitierte Arbeit von Langstein - Edelstein hin.

1) Burow, Baseler Diss. 1905. Zit. nach Sommerfeld, Milchkunde. Wiesbaden 1909. S. 165.

2) Langstein-Edelstein, Über die Einheitlichkeit des Frauenmilchcaseins. Jahrb, f. Kinderheilk. 72, Ergänzungsheft, S. 1 ff. 1910. 
Es ist eine altbekannte Tatsache, daß das Frauenmilchcasein sich beim Ausfällen aus der Milch ganz anders verhält als z. B. Kuhmilchcasein. Dies kann entweder auf der Verschiedenheit des Frauenmilchcaseins selbst oder auf Milieuverschiedenheiten oder auch auf beiden beruhen. Durch die Bestimmung des isoelektrischen Punktes einiger Caseinarten versuchten wir dieser Frage näher zutreten.

Als den isoelektrischen Punkt eines Eiweißkörpers bezeichnet man nach Michalis ${ }^{1}$ ) diejenige Wasserstoffionenkonzentration, bci der das Eiweiß isoelektrisch ist, d. h. die elektrische Ladung gleich Null ist, und wo also das Eiweiß bei kataphoretischen Uberführungsversuchen weder anodisch noch kathodisch wandert.

Michaelis hat in mehreren Arbeiten nachgewiesen, daß der isoelektrische Punkt der kolloidalen Ampholyte, unter anderem des Kuhcasein ${ }^{2}$ ), mit dem Flockungsoptimum der betreffenden Ampholyte zusammenfällt. Danach wird die Bestimmung des isoelektrisehen Punktes bedeutend vereinfacht: statt elektrische Uberführungsversuche zu machen, bestimmt man das Flockungsoptimum, und wenn es einmal gefunden worden ist, mißt man elektrometrisch, durch die Gaskettenmethode, die $\left[\mathrm{H}^{*}\right]$ im Optimum; der gefundene Wert ist dann der Wert des isoelektrischen Punktes.

In den folgenden Bestimmungen haben wir dieselbe Technik wie Michaelis und Pechstein'2), angewandt.

In den Tabellen, wo die einzelnen Flockungsversuche angeführt sind, sieht man die Mengen und Konzentrationen der angewandten Casein-, Acetat- und Essigsäurelösungen.

0 bedeutet $=$ klar $;+=$ Trübung; $+=$ stärkere Trübung und $x=$ Flockung.

Wir hatten Frauenmilch, Kuhmilch, Ziegenmilch, Hundemilch und Milch von Meerschweinchen zur Verfügung. Von allen diesen wurde Casein auf folgende Weise dargestellt (s. Darstellung nach LangsteinEdelstein, 1. c. S. 14: Frische Milch wurde zuerst ca. 1 Stund ezentrifugiert, das Fett abgeschöpft, mit der 4 fachen Menge Wasserleitungswasser verdünnt und dann mit $1 / 5$ des Gesamtvolumens $\frac{\mathrm{n}}{10}$ Essigsäure unter Umrühren versetzt, das abgesetzte Casein mit destilliertem

1) Vgl. darüber L. Mich a e lis, Die allgemeine Bedeutung der Wasserstoffionenkonzentration für die Biologie; in Oppenheimers Handbuch der Biochemie, Ergänzungsband (1913).

2) Michaelis und Pechstein, Biochem. Zeitschr. 47, S, $260 \mathrm{ff}$. 
Wasser, Alkohol und Äther ausgewaschen, pulverisiert und ohne zu trocknen zu Versuchen angewandt.

Bei der Darstellung von Meerschweinchenmilchcasein fiel eigentümlicherweise nach ea. 15 Minuten Zentrifugieren ein dicker, körniger Niederschlag aus; die Flüssigkeit oben blieb milchig opalescierend. Die Flüssigkeitsschicht wurde abpipettiert, mit Essigsäure versetzt, dabei fiel nur eine geringe Menge von feinen Flocken aus. Der körnige Niederschlag wurde wie oben ausgewaschen. Auf diese Weise bekamen wir von $9,0 \mathrm{ccm}$ Milch $0,28 \mathrm{~g}$ von dem beim Zentrifugieren ausgefallenen Körper, während der mit Essigsäure ausgefällte Körper nur 0,04 g wog. Nachher zeigte sich, daß diese beiden Körper Casein waren und beide denselben isoelektrischen Punkt hatten.

\section{Kuhmilcheasein.}

Zuerst wurde der isoelektrische Punkt eines von uns dargestellten Kuhmilchcaseins bestimmt, dann der eines im Handel erhältlichen Kuhmilchcaseins und zuletzt der isoelektrische Punkt einer Probe von unserem Kuhmilchcasein, das 24 Stunden im Thermostat bei $56^{\circ} \mathrm{C}$ (mit $\frac{\mathrm{n}}{\mathrm{I}}$ Natriumacetat versetzt) gestanden hatte. Wie aus folgenden Tabellen zu ersehen, bekamen wir in allen diesen Fällen Werte, die ganz gut übereinstimmten mit dem früher von Michaelis und Pech stein gefundenen Mittelwert 2,5 $10^{-5}$ des isoelektrischen Punktes für Kuhmilcheasein. Verunreinigungen und Hydrolyseprodukte stören also bei dieser Verdünnung, die wir angewandt haben, gar nicht die Bestimmung des isoelektrischen Punktes.

Versuch 1. Kuhmilchcasein, selbst dargestelltes Präparat, $0,1 \mathrm{~g}$, unter leichtem Erwärmen in $15 \mathrm{ccm} \frac{\mathrm{n}}{\mathrm{l}}$ Natriumacetat gelöst, auf $150 \mathrm{ccm}$ mit destilliertem $\mathrm{H}_{2} \mathrm{O}$ aufgefüllt.

\begin{tabular}{|c|c|c|c|c|c|c|c|c|c|}
\hline Röhrchea & 1 & 2 & 3 & 4 & 5 & 6 & 7 & 8 & $\mathbf{g}$ \\
\hline Wasser.......... & 8,64 & 8,50 & 8,25 & 7,88 & 7,31 & 6,56 & 5,2 & 3,3 & 0,74 \\
\hline 0,07 proz. Casein in $\frac{\mathrm{n}}{10}$ Acetat & 1,0 & 1,0 & 1,0 & 1,0 & 1,0 & 1,0 & 1,0 & 1,0 & 1,0 \\
\hline$\frac{\mathrm{n}}{10}$ Essigsäure $\ldots \ldots$. & 0,36 & 0,5 & 0,75 & 1,13 & 1,69 & 2,44 & 3,8 & 5,7 & 8,26 \\
\hline $\begin{array}{l}\text { Nach } 5 \text { Minuten } \\
20\end{array}$ & 0 & + & $\begin{array}{l}+ \\
+1\end{array}$ & $H$ & it & $\begin{array}{l}+ \\
+(?)\end{array}$ & $t$ & $\begin{array}{l}+ \\
+\end{array}$ & $\begin{array}{r}0 \\
+\end{array}$ \\
\hline$" 24$ Stunden & 0 & $x \times$ & $x \times$ & $\times x$ & $x \times$ & $\pi x$ & H & + & + \\
\hline
\end{tabular}


Nach 24 Stunden total ausgeflockt Röhrchen 2-6; Mittel Röhrchen 4 , dessen $\left[\mathrm{H}^{\circ}\right]$ nach der Formel $\left.{ }^{1}\right)\left[\mathrm{H}^{*}\right]=\frac{1,86 \cdot 10^{-5}}{\alpha} \cdot \frac{\text { Essigsäure }}{\text { Acetat }}$ berechnet $(\alpha$ hier $=0,79):\left[\mathrm{H}^{*}\right]=2,5 \cdot 10^{-5}$.

Versuch 2. Technisches Kuhmilchcasein; die Lösung ganz wie im Versuch 1 .

\begin{tabular}{|c|c|c|c|c|c|c|c|c|c|}
\hline Röhrehen & 1 & 2 & 3 & 4 & 5 & 6 & 7 & 8 & 9 \\
\hline Wasser ............ & 8,64 & 8,50 & 8,25 & 7,88 & 7,31 & 6,56 & 5,2 & 3,3 & 0,75 \\
\hline 0,07 proz. Cascin in $\frac{n}{10}$ Acetat & 1,0 & 1,0 & 1,0 & 1,0 & 1,0 & 1,0 & 1,0 & 1,0 & 1,0 \\
\hline$\frac{\mathrm{n}}{10}$ Essigsäure . . . . . & 0,36 & 0,5 & 0,75 & 1,13 & 1,69 & 2,44 & 3,8 & 5,7 & 8,26 \\
\hline $\begin{array}{c}\text { Nach } 10 \text { Minuten... } \\
" 50 \quad, \quad \ldots\end{array}$ & $\begin{array}{l}0 \\
+\end{array}$ & $\begin{array}{l}0 \\
+\end{array}$ & + & $\begin{array}{l}+1 \\
x \\
\end{array}$ & $\begin{array}{l}+ \\
x\end{array}$ & $\begin{array}{l}+1+8 \\
+\end{array}$ & $\begin{array}{l}+ \\
+\end{array}$ & $\begin{array}{l}0 \\
+\end{array}$ & 0 \\
\hline
\end{tabular}

Wie oben berechnet: Mittel Röhrchen $4:\left[\mathrm{H}^{\circ}\right]=2,5 \cdot 10^{-5}$.

Versuch 3. Kuhmilchcasein; dasselbe Präparat wie im Versuch 1. $0,1 \mathrm{~g}$ mit $10 \mathrm{ccm} \frac{\mathrm{n}}{1}$ Natriumacetat 24 Stunden im Thermostaten $56^{\circ} \mathrm{C}$ gestanden, dann auf $100 \mathrm{ccm}$ mit destilliertem $\mathrm{H}_{2} \mathrm{O}$ gefüllt.

\begin{tabular}{|c|c|c|c|c|c|c|c|c|c|}
\hline Röhrchen & 1 & 2 & 3 & 4 & 5 & 6 & 7 & 8 & 9 \\
\hline Wasser.......... & 8,5 & 8,33 & 8,12 & 7,83 & 7,44 & 6,96 & 6,23 & 5,32 & 4,1 \\
\hline 0,1 proz. Casein in $\frac{n}{10}$ Acetat & 1,0 & 1,0 & 1,0 & 1,0 & 1,0 & 1,0 & 1,0 & 1,0 & 1,0 \\
\hline$\frac{n}{10}$ Essigsäure . . . . . & 0,5 & 0,67 & 0,88 & 1,17 & 1,56 & 2,04 & 2,77 & 3,68 & 4,9 \\
\hline $\begin{array}{c}\text { Nach } 10 \text { Minuten } \ldots \ldots \\
\Rightarrow \quad 30 \quad n \quad \ldots \ldots \\
" \quad 1 \text { Stunde } \ldots \ldots\end{array}$ & $\begin{array}{l}0 \\
0 \\
0\end{array}$ & $\begin{array}{l}+ \\
+i \\
+i\end{array}$ & $\begin{array}{l}+ \\
(x) \\
x\end{array}$ & $\begin{array}{l}+ \\
(x) \\
x\end{array}$ & $\begin{array}{l}+ \\
x \\
x\end{array}$ & $\begin{array}{c}+4 \\
(x) \\
x\end{array}$ & $\begin{array}{c}+ \\
(x) \\
x\end{array}$ & $\mid \begin{array}{c}+ \\
(\times ?) \\
(\times ?)\end{array}$ & $\begin{array}{l}+ \\
+ \\
\times ?)\end{array}$ \\
\hline
\end{tabular}

Mittel Röhrchen 5; in demselben elektrometrisch gemessen: $\left[\mathrm{H}^{\prime}\right]=2,6 \cdot 10^{-5}$.

Versuch 4.. Alles wie im Versuch 3. Optimale Ausflockung im Röhrchen 5; elektrometrisch gemessen: $\left[\mathrm{H}^{\circ}\right]=2,7 \cdot 10^{-5}$.

\section{Ziegenmilcheasein.}

Versuch $1.0,1 \mathrm{~g}$ beim leichten Erwärmen in $15 \mathrm{ccm} \frac{\mathrm{n}}{1}$ Natriumacetat gelöst, auf $150 \mathrm{ccm}$ gefüllt.

1) Michaelis, in Abderhaldens Biochem. Arbeitsmethoden 3, S. 1341 und $5 \mathrm{a}, \mathrm{S} .524$. 


\begin{tabular}{|c|c|c|c|c|c|c|c|c|c|}
\hline Röhroben & 1 & 2 & 3 & 4 & 5 & 6 & 7 & 8 & 9 \\
\hline Wasser........... & 8,64 & 8,5 & 8,25 & 7,88 & 7,31 & 6,56 & 5,2 & 3,3 & 0,74 \\
\hline 0,07 proz. Casein in $\frac{\mathrm{n}}{10}$ Acetat & 1,0 & 1,0 & 1,0 & 1,0 & 1,0 & 1,0 & 1,0 & 1,0 & 1,0 \\
\hline${ }_{10}^{\mathrm{n}}$ Essigsäure $\ldots \ldots$. & 0,36 & 0,5 & 0,75 & 1,13 & 1,69 & 2,44 & 3,8 & 5,7 & 8,26 \\
\hline Nach 10 Minuten. & 0 & + & + & + & $\cdots$ & + & + & 0 & 0 \\
\hline$n \quad 50 \quad n$ & 0 & $\because$ & $x$ & $x$ & $x$ & $+(++?)$ & + & + & 0 \\
\hline
\end{tabular}

Optimale Ausflockung in Röhrchen 4, dessen H-Ionenkonzontration wie oben berechnet: $\left[\mathrm{H}^{\top}\right]=2,5 \cdot 10^{-3}$.

Versuch 2. Casein von Ziegencolostrum (2 Stunden vor der Geburt gemolken). In Lösung gebracht und verdünnt ganz wie im Versuch 1 .

\begin{tabular}{|c|c|c|c|c|c|c|c|c|c|}
\hline Röhrchen & 1 & 2 & 3 & 4 & 5 & 6 & 7 & 8 & 9 \\
\hline Wasser. . & 8,5 & 8,33 & 8,12 & 7,83 & 7,44 & 6,96 & 6,23 & 5,32 & 4,1 \\
\hline 0,07 proz. Casein in $\frac{n}{10}$ Acetat & 1,0 & 1,0 & 1,0 & 1,0 & 1,0 & 1,0 & 1,0 & 1,0 & 1,0 \\
\hline$\frac{10}{n}$ Essigsäure & 0,5 & 0,67 & 0,88 & 1,17 & 1,56 & 2,04 & 2,77 & 3,68 & 4,9 \\
\hline $\begin{array}{cccc}\text { Nach } & 10 & \text { Minuten } & \ldots \\
, & 1 & \text { Stunde } \ldots \\
\end{array}$ & 0 & + & $x x$ & $x \times$ & $\therefore x$ & $x \times$ & $x$ & $+(?)$ & $\begin{array}{l}+ \\
+\end{array}$ \\
\hline
\end{tabular}

Optimale Ausflockung in Röhrchen 5, dessen H-Ionenkonzentration elektrometrisch gemessen $=2,6 \cdot 10^{-5}$.

Versuch 3. Ziegenmilchcasein, $0,1 \mathrm{~g}$, mit $10 \mathrm{ccm} \frac{\mathrm{n}}{\mathrm{l}}$ Natriumacetat versetzt, 24 Stunden im Thermostaten $56^{\circ} \mathrm{C}$ gestanden, dann auf $100 \mathrm{ccm}$ mit $\mathrm{H}_{2} \mathrm{O}$ gefüllt.

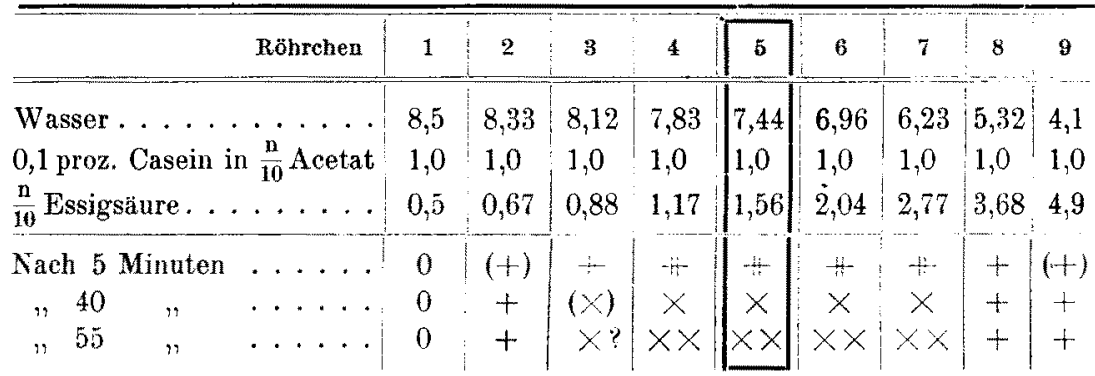

Optimale Ausflockung in Röhrchen 5, H-Ionenkonzentration elektrometrisch gemessen: $\left[\mathrm{H}^{\circ}\right]=2,6 \cdot 10^{-5}$.

In allen Versuchen sind die Reihen hier ganz ähnlich denjenigen, die wir bei Kuhmilchcasein getroffen haben: die Asymmetrie der 
Reihen nach rechts sowie der isoelektrische Punkt stimmen ganz mit denen des Kuhmilchcaseins überein. Die Asymmetrie ist eine Erscheinung der Flockungsreihen, die von L. Michaelis und Rona, später genauer von Michaelis und Pechstein (l. c.) beschrieben wurde, und welche in folgendem besteht. Setzt man die Reihen stets so an, daß die Wasserstoffionenkonzentration in geometrischer Progression ansteigt, und faßt man nun das Flockungsoptim um ins Auge, so bemerkt man, daß die Abnahme der Flockung und Trübung nach rechts (also mit steigender $\mathbf{H}^{-}$-Konzentration) allmählicher, unschärfer stattffndet als nach links (mit fallender $\mathrm{H}^{*}$-Konzentration).

\section{Hundemilehcasein.}

Versuch 1. 0,1 g mit $10 \mathrm{~cm}{ }_{1}^{\mathrm{n}}$ Natriumacetat versetzt, leicht erwïrmt, auf $100 \mathrm{cem}$ mit $\mathrm{H}_{2} \mathrm{O}$ gefüllt.

\begin{tabular}{|c|c|c|c|c|c|c|c|c|c|c|c|}
\hline Röhrchen & 1 & 2 & 3 & 4 & 5 & 6 & 7 & 8 & 9 & 10 & 11 \\
\hline 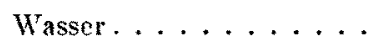 & 8,62 & $8, \bar{y}$ & 8,33 & 8,12 & 7,83 & 7,44 & 6,96 & 6,28 & 5,32 & 4,1 & 2,5 \\
\hline 0,1 proz. Casein in $\frac{n}{10}$ Acetat & 1,0 & 1,0 & 1,0 & 1,0 & 1,0 & 1,0 & 1,0 & 1,0 & 1,0 & 1,0 & 1,0 \\
\hline$\frac{\mathrm{n}}{10}$ Essigsäure . . . . . & 0,38 & 0,5 & 0,67 & 0,88 & 1,17 & 1,56 & 2,04 & 2,77 & 3,68 & 4,9 & 6,5 \\
\hline Nach 10 Minuten & 0 & + & $\cdots$ & $\therefore$ & $+\cdots$ & $\because$ & + & + & + & + & 0 \\
\hline$" 50$ & 0 & + & $x$ & x & $x$ & x & $x$ & $(x)$ & + & + & + \\
\hline
\end{tabular}

Versuch 2. Gleiche Anordnung wie im Versuch 1.

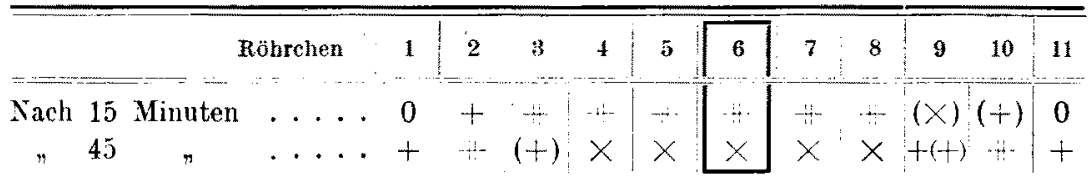

Im Versuch I war das Ausflockungsoptimum in Röhrchen 5; HIonenkonzentration gemessen: $\left[\mathrm{H}^{*}\right]=2,15 \cdot 10^{-5}$.

Im Versuch 2 war Optimum im Röhrchen 6, und H-Ionenkonzentration gemessen: $\left[\mathrm{H}^{\circ}\right]=3,2 \cdot 10^{-5}$.

Im Mittel: $\left[\mathrm{H}^{\circ}\right]=2,7 \cdot 10^{-5}$.

Also auch Hundecasein hat denselben isoelektrischen Punkt und zeigt dieselbe Asymmetrie nach rechts wie Kuh- und Ziegenmilchcasein.

\section{Meerschweinchenmileheasein.}

Versuch 1. 0,1 g von dem beim Zentrifugieren ausgefallenen Körper mit $10 \mathrm{~cm} \frac{\mathrm{n}}{1}$ Natriumacetat leicht erwärmt, auf $100 \mathrm{~cm} \mathrm{mit} \mathrm{H}_{2} \mathrm{O}$ aufgefüllt. 


\begin{tabular}{|c|c|c|c|c|c|c|c|c|c|}
\hline Röhrehen & 1 & 2 & 3 & 4 & 5 & 6 & 7 & 8 & 9 \\
\hline \multirow{2}{*}{$\begin{array}{l}\text { Wasser . . . . . . . . } \\
0,1 \text { proz. Casein in } \frac{\mathrm{n}}{10} \text { Acetat }\end{array}$} & 8,0 & 7,0 & 5,0 & 1,0 & 7,4 & 5,8 & 2,6 & 7,72 & 6,44 \\
\hline & 1,0 & 1,0 & 1,0 & 1,0 & 1,0 & 1,0 & 1,0 & & 1,0 \\
\hline \multirow[t]{2}{*}{ Essigsäure . } & $\frac{n}{100}$ & $\frac{n}{100}$ & $\frac{n}{100}$ & $\frac{n}{100}$ & $\frac{\mathrm{n}}{10}$ & $\frac{\mathrm{n}}{10}$ & $\frac{\mathrm{n}}{10}$ & $\frac{n}{1}$ & $\frac{n}{1}$ \\
\hline & 1,0 & 2,0 & 4,0 & 8,0 & 1,6 & 3,2 & 6,4 & 1,28 & 2,56 \\
\hline Nach 10 Minuten & 0 & 0 & 0 & $H$ & $+(+)$ & $+(+)$ & + & 0 & 0 \\
\hline$\Rightarrow \quad 30$ & 0 & 0 & + & $x \times$ & $x(x)$ & $+(+)$ & + & + & +0 \\
\hline
\end{tabular}

Optimale Ausflockung in Röhrchen 4; H-Ionenkonzentration gemessen: $\left[\mathrm{H}^{*}\right]=1,9 \cdot 10^{-5}$.

Versuch 2. $0,04 \mathrm{~g}$ von dem mit Essigsäure ausgefällten Körper wurden mit $5,0 \mathrm{ccm} \frac{\mathrm{n}}{\mathrm{l}}$ Natriumacetat leicht erwärmt, auf $50 \mathrm{ccm}$ mit $\mathrm{H}_{2} \mathrm{O}$ gefüllt.

\begin{tabular}{|c|c|c|c|c|c|c|c|c|c|}
\hline Röhrchen & 1 & 2 & 3 & 4 & 5 & 6 & 7 & 8 & 9 \\
\hline Wasser... & 8,5 & 8,33 & 8,12 & 7,83 & 7,44 & 6,96 & 6,23 & 5,32 & 4,1 \\
\hline 0,08 proz. Casein . . . . & 1,0 & 1,0 & 1,0 & 1,0 & 1,0 & 1,0 & 1,0 & 1,0 & 1,0 \\
\hline$\frac{\mathrm{n}}{10}$ Essigsäure $\ldots$. & 0,5 & 0,67 & 0,88 & 1,17 & 1,56 & 2,04 & 2,77 & 3,68 & 4,9 \\
\hline Nach 20 Minuten & $(+)$ & + & 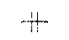 & + & + & + & + & + & + \\
\hline
\end{tabular}

Das Optimum in Röhrchen 4, H-Ionenkonzentration gemessen: $\left[\mathrm{H}^{*}\right]=2,2 \cdot 10^{-5}$.

Die gefundenen Werte $1,9 \cdot 10^{-5}$ und $2,2 \cdot 10^{-5}$ differieren so wenig voneinander, daß die Differenz ganz gut innerhalb der methodischen Fehlergrenzen liegen kann; und dabei ist noch zu beachten, daß im Versuch 1 die Essigsäuremengen in zwei einander folgenden Röhrchen wie $1: 2$ sich verhielten, während wir in allen anderen Versuchen feinere Abstufungen und ein Verhältnis $1: 1 \frac{1}{3}$ hatten. Durch diese Anordnung wurde im Versuch 1 die Abgrenzung des isoelektrischen Punktes unschärfer. Die Reihen der beiden Körper gleichen einander ganz, beide zeigen schön diese eigentümliche, nach Michaelis ${ }^{1}$ ) auf Salzwirkung beruhende Asymmetrie, die wir schon bei den vorigen Caseinen getroffen haben.

Ohne Bedenken können wir also die beiden Körper als identisch und als Casein betrachten.

Den Mittelwert des isoelektrischen Punktes von Meerschweinchenmilchcasein fanden wir demnach $\mathrm{zu}:\left[\mathrm{H}^{\top}\right]=2,1 \cdot 10^{-5}$.

1) Michaelis und Pechstein, Biochem. Zeitschr. 47, S. $260 \mathrm{ff}$. 
Bei den vorigen Caseinen fanden wir Werte von $2,5-2,7 \cdot 10^{-5}$. Die Differenz, die der gefundene isoelektrische Punkt zeigt, ist zu klein, um etwas Bestimmtes für die Verschiedenheit des Meerschweinchenmilcheaseins sagen zu können.

Zur oben beschriebenen eigentümlichen Fällbarkeit des Meerschweinchenmilchcaseins kann die Lage des isoelektrischen Punktes keine Erklärung geben; sie muß also auf irgendeiner besonderen Milieuverschiedenheit beruhen.

\section{Frauenmilchcasein.}

Wir hatten 3 verschiedene Präparate: ein von uns dargestelltes, ein Präparat nach Engel und eins nach Langstein-Edelstein. Von jedem dieser wurden mehrere Reihen angestellt; in folgenden Tabellen werden nur diejenigen Versuche angegeben, bei denen [ $\left.\mathrm{H}^{*}\right]$ elektrometrisch gemessen wurde.

Versuch 1. Präparat Langstein - Edelstein: 0,1 g mit $10 \mathrm{~cm}$ $\frac{n}{2}$ Natriumacetat leicht erwärmt, auf $50 \mathrm{~cm}$ mit $\mathrm{H}_{2} \mathrm{O}$ gefüllt, von Ungelöstem abfiltriert:

\begin{tabular}{|c|c|c|c|c|c|c|c|c|c|c|}
\hline Röhrchen & 1 & 2 & $\mathbf{3}$ & 4 & 5 & 6 & 7 & 8 & 9 & 10 \\
\hline Wasser $\ldots \ldots \ldots$ & 8,5 & 8,33 & 8,12 & 7,83 & 7,44 & 6,96 & 6,23 & 5,32 & 4,1 & 2,5 \\
\hline C. 0,1 proz. Casein in $\frac{n}{10}$ Acetat & 1,0 & 1,0 & 1,0 & 1,0 & 1,0 & 1,0 & 1,0 & 1,0 & 1,0 & 1,0 \\
\hline$\frac{\mathrm{n}}{10}$ Essigsiiure . . . . & 0,5 & 0,67 & 0,88 & 1,17 & 1,56 & 2,04 & 2,77 & 3,68 & 4,9 & 6,5 \\
\hline $\begin{array}{l}\text { Nach } 5 \text { Minuten } \\
" 30 "\end{array}$ & 0 & 0 & $\begin{array}{l}0 \\
+\end{array}$ & $\begin{array}{c}(+) \\
+\end{array}$ & $\begin{array}{l}+ \\
+\end{array}$ & $\begin{array}{l}H \\
H\end{array}$ & $\begin{array}{c}+(t) \\
+\end{array} \mid$ & $(+)$ & & \\
\hline
\end{tabular}

Versuch 2. Dasselbe Präparat. Gleiche Anordnung wie im Versuch 1 .

\begin{tabular}{ccc|c|c|c|c|c|c|c|c|c|c}
\hline & Röhrohen & 1 & 2 & 3 & 4 & 5 & 6 & 7 & 8 & 9 & 10 \\
\hline Nach 5 Minuten & $\ldots$ & $\ldots$ & 0 & 0 & $(+)$ & + & + & + & + & 0 & 0 & + \\
$n 30$ & $n$ & $\ldots$ & $\ldots$ & & $(+)$ & + & $+(+)$ & + & + & + & 0 & 0
\end{tabular}

Versuch 3. Dasselbe Präparat. Gleiche Anordnung wie in Versuchen 1 und 2 .

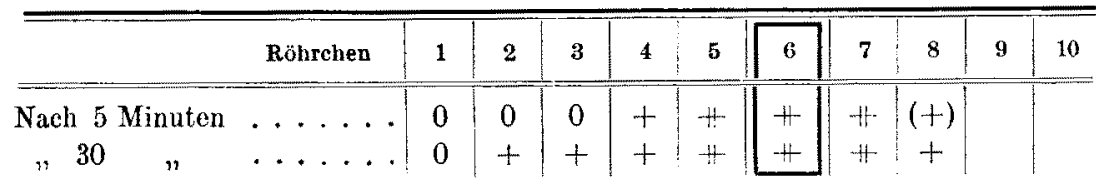


Das Ausflockungsoptimum war in allen Versuchen im Röhrchen 6, und gemessen:

$$
\begin{array}{r}
\text { Versuch 1: }\left[\mathrm{H}^{\circ}\right]=3,6 \cdot 10^{-5} \\
\text { Versuch } 2:\left[\mathrm{H}^{\dagger}\right]=5,0 \cdot 10^{-5} \\
\text { Versuch } 3:\left[\mathrm{H}^{\circ}\right]=4,9 \cdot 10^{-5} \\
\text { Mittel: }\left[\mathrm{H}^{*}\right]=4,5 \cdot 10^{-5} .
\end{array}
$$

Versuch 1. Eigenes Präparat. $0,1 \mathrm{~g}$ wurde mit $10 \mathrm{ccm} \frac{\mathrm{n}}{2} \mathrm{Natrium-}$ acetat leicht erwärmt, auf $50 \mathrm{ccm}$ mit $\mathrm{H}_{2} \mathrm{O}$ gefüllt, ein beträchtlicher Teil blieb ungelöst.

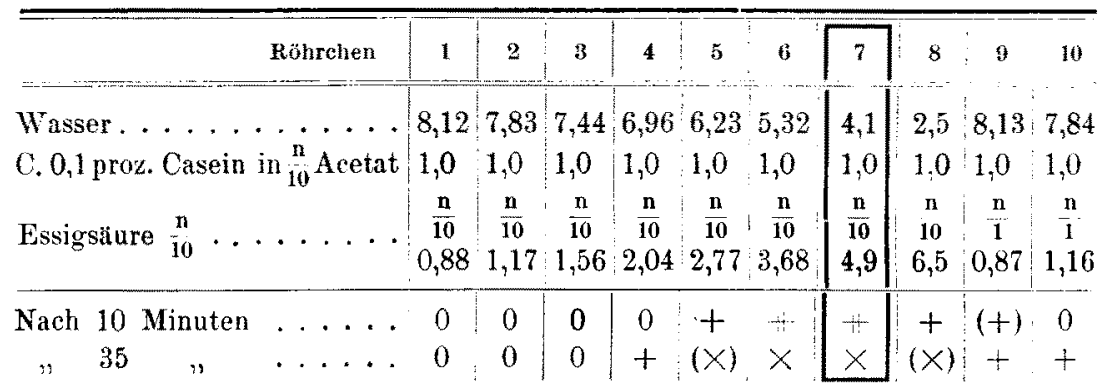

Versuch 2. Gleiche Anordnung wie im Versuch 1.

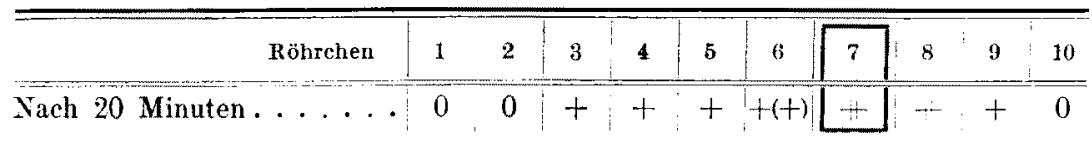

Versuch 3. Gleiche Anordnung wie in Versuchen 1 und 2.

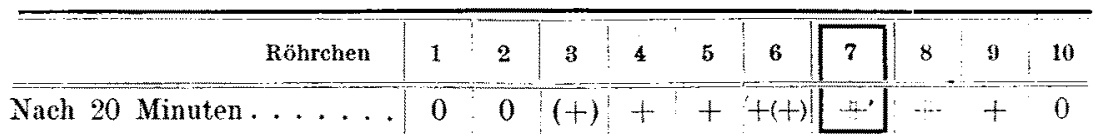

Das Flockungsoptimum in allen Versuchen im Röhrchen 7, und gemessen:

$$
\begin{array}{r}
\text { Versuch 1: }\left[\mathrm{H}^{*}\right]=7,9 \cdot 10^{-5} . \\
\text { Versuch 2: }\left[\mathrm{H}^{*}\right]=7,9 \cdot 10^{-5} . \\
\text { Versuch } 3:\left[\mathrm{H}^{\circ}\right]=7,9 \cdot 10^{-5} . \\
\text { Mittel: }\left[\mathrm{H}^{*}\right]=7,9 \cdot 10^{-5} .
\end{array}
$$

Präparat nach Engel. $0,1 \mathrm{~g}$ mit $5 \mathrm{ccm} \frac{\mathrm{n}}{2}$ Natriumacetat (= halbe Salzmenge!) leicht erwärmt, bis $50 \mathrm{ccm}$ mit $\mathrm{H}_{2} \mathrm{O}$ gefüllt, filtriert. 


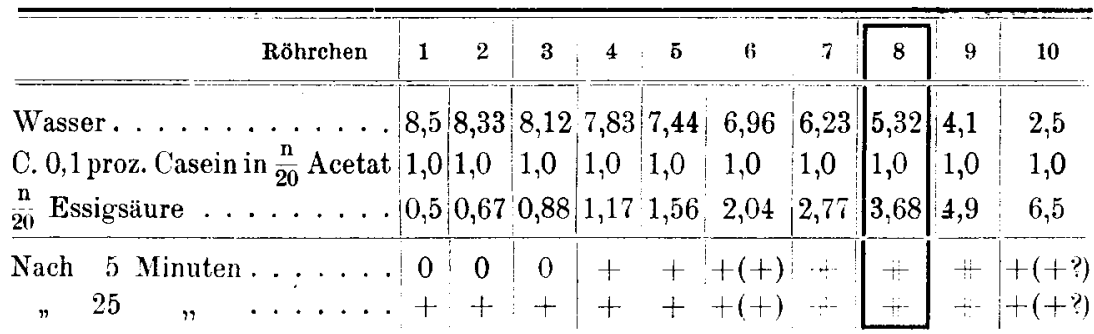

Das Flockungsoptimum war im Röhrchen 8, elektrometrisch gemessen: $\left[\mathrm{H}^{\circ}\right]=8,2 \cdot 10^{-5}$.

Betrachten wir jetzt näher die gefundenen Werte für das Frauenmilchcasein, so sehen wir, daß der isoelektrische Punkt in merkbar saurerem Gebiete liegt als bei den vorigen Caseinen. Die Werte der verschiedenen Frauenmilchcaseinpräparate schwanken in Grenzen zwischen 4,5-8,2, was sich aber nicht durch den Reinheitsgrad der verschiedenen Präparate erklären läßt, wie dies die Versuche mit Kuhmilchcasein zeigen, sondern wohl die Fehlergrenzen der Methode darstellt.

Nehmen wir nun den Mittelwert von den verschiedenen Präparaten, so bekommen wir für das Frauenmilchcasein: $\left[\mathrm{H}^{\prime}\right]=6,9 \cdot 10^{-5}$.

Der isoelektrische Punkt des Frauenmilchcaseins liegt also in einem deutlich saurerem Gebiete als der der anderen untersuchten Caseine. Wenn wir noch die einzelnen Reihen des Frauenmilchcaseins betrachten, so bemerken wir, daß die Asymmetrie hier ein ganz anderes Verhältnis zeigt als bei den anderen Caseinen. Während dort das Flockungsgebiet sich regelmäßig bedeutend weiter nach rechts vom Koagulationsoptimum erstreckte, nehmen hier die Trübungen nach links allmählicher ab.

Nach alledem läßt sich ohne Zweifel sagen, daß wir im Frauenmilchcasein mit einem von Kuh-, Ziegen-, Hunde- und Meerschweinchenmilchcasein verschiedenen Körper zu tun haben. Näher gesagt, besteht dieser Unterschied darin, daß das Frauenmilchcasein im Vergleich zu den anderen untersuchten Caseinen einen ausgeprägteren Säurecharakter hat. Dieses läßt sich mit Hilfe der Dissoziationstheorie und der Theorie des isoelektrischen Punktes auf folgende Weise ableiten:

Caseine, als amphotere Eiweißkörper, können nach folgenden Gleichungen dissoziiert werden:

$$
\begin{aligned}
& \mathrm{H} \cdot \text { Casein } \cdot \mathrm{OH}=\mathrm{H} \cdot \text { Casein }^{+}+\mathrm{OH}^{-} \text {(als Base) und } \\
& \mathrm{H} \cdot \text { Casein } \cdot \mathrm{OH}=\mathrm{H}^{+}+\mathrm{Casein}^{-\mathrm{OH}^{-}} \text {(als Säure). }
\end{aligned}
$$


Um nun die maximale Ausflockung des Caseins oder, was dasselbe ist, den isoelektrischen Zustand hervorzurufen, muß

sein.

$$
\left[\mathrm{H} \cdot \text { Casein }^{+}\right]=\left[\text {Casein } \cdot \mathrm{OH}^{-}\right]
$$

Bei dem Frauenmilchcasein mußten wir eine höhere $\left[\mathrm{H}^{*}\right]$ haben, um zur maximalen Ausflockung zu kommen. Dies wieder berleutet, da $B$ das Frauenmilchcasein unter gleichen Arbeitsbedingungen einen stärkeren Säurecharakter hat als die anderen untersuchten Caseine.

\section{Zusammenfassung.}

Das Ausflockungsoptimum und damit der isoelektrische Punkt des Ziegenmilch- und Ziegencolostrumcaseins (bei beiden gefunden [ $\left.\mathrm{H}^{\cdot}\right]$ $\left.=2,6 \cdot 10^{-5}\right)$, des Hundemilcheaseins $\left(\left[\mathrm{H}^{-}\right]=2,7 \cdot 10^{-5}\right)$ und wahrscheinlich auch des Meerschweinchenmilchcaseins $\left(\left[\mathrm{H}^{*}\right]=2,1 \cdot 10^{-5}\right)$ fallen zusammen mit dem des Kuhmilchcaseins $\left(\left[\mathrm{H}^{*}\right]=2,6 \cdot 10^{-5}\right)$. Alle diese Caseine zeigen auch eine ganz gleiche Asymmetrie der Flockungsreihen nach rechts. Das Frauenmilchcasein dagegen zeigt eine Asymmetrie nach links, und sein isoelektrischer Punkt liegt in höherer $\left[\mathrm{H}^{\circ}\right]$ $=6,9 \cdot 10^{-5}$, was darauf deutet, daß das Frauenmilchcasein im Vergleich zu den anderen untersuchten Caseinen einen stärkeren Säurecharakter haben muß.

Zum Schluß möchte ich noch meinen besten Dank Herrn Prof. Leonor Michaelis sagen, der mich in liebenswürdiger Weise in die Methodik eingeführt hat. 